\title{
Chest Radiograph Abnormalities in Patients Hospitalized with Leptospirosis in the City of Salvador, Bahia, Brazil
}

\author{
Eliana Dias Matos, Everaldo Costa, Edilson Sacramento, \\ Anna Luiza Caymmi, César de Araújo Neto, \\ Marcelo Barreto Lopes and Antonio Alberto Lopes
}

\author{
Octávio Mangabeira Hospital, Bahia School \\ of Medicine and Public Health, Couto Maia \\ Hospital, São Rafael Hospital, Federal \\ University of Bahia, Salvador, Bahia, Brazil
}

\begin{abstract}
This study was designed to estimate the prevalence of pulmonary radiograph abnormalities and describe the distribution of the patterns of radiographic alterations among patients hospitalized with leptospirosis. Chest radiographs of 139 patients hospitalized with leptospirosis in Couto Maia Hospital, in Salvador, Bahia, Brazil, between July, 1997, and July, 1999, were analyzed. The radiographs were requested soon after hospital admission, independent of the clinical manifestations of the patients. Only the first radiograph was considered. Pulmonary radiograph alterations were recorded in $35 / 139$ patients $(25.2 \%)$; $95 \%$ mid-point confidence interval $=$ $18.5 \%$ to $32.9 \%$. Among the patients with radiograph alterations, alveolar infiltrate was seen in $26 / 35(74.3 \%)$. The lesions were bilateral in $54.3 \%$ and located in the inferior lobes in $45.5 \%$. Pleural effusion, represented by blunting of the costo-phrenic angle, was detected in $8.6 \%$ of the patients. The pattern of the pulmonary alterations, predominantly bilateral alveolar infiltrates, is consistent with the evidence that the basic pulmonary alteration in leptospirosis is a generalized capillaritis.

Key Words: Leptospirosis, chest radiography.
\end{abstract}

Leptospirosis is a zoonotic disease prevalent worldwide, but with they highest incidence in tropical countries [1-7]. In Brazil, this disease is endemic in several regions with outbreaks occurring in relation to seasons of increased precipitation and contact with flood waters contaminated with urine of infected animals, particularly rats $[7,8]$. Clinical manifestations of the disease are the result of a multisystemic involvement [9]. In spite of the potential importance of

Received on 10 December 2000; revised 12 April 2001.

Address for correspondence: Dr. Antonio Alberto Lopes, MD, MPH, PhD. Rua Mar. Floriano 448 apt 1301, Canela, Zip Code: 40110-010, Salvador - Bahia, Brazil; Phone: 55 71-3361558. Fax: 55 71-2457110.E-mail: aaslopes@ufba.br This work was developed at the Clinical Epidemiology Unit of the Edgard Santos Hospital, Federal University of Bahia and Couto Maia Hospital of the Health Department of the State of Bahia

The Brazilian Journal of Infectious Diseases 2001;5(2):73-77 (C) 2001 by The Brazilian Journal of Infectious Diseases and Contexto Publishing. All rights reserved. $1413-8670$ lung involvement in leptospirosis, few studies have adequately addressed questions related to the pulmonary radiographic alterations of the disease.

Estimates of frequency of pulmonary radiographic alterations in leptospirosis have varied from $11 \%$ to $67 \%[1-6,10,11]$. This great variation may be partly related to the criteria used to request radiographic exams and the lack of precision of the estimates due to a relatively small sample size. However, the possibility that this variation is related to differences in the distribution of various serotypes of leptospira across geographic regions, cannot be completely ruled out. It is important to note, that the highest frequencies of radiographic alterations have been reported in studies from Korea, China and Reunion Island $[1,2,4,11]$. By contrast, studies from the western hemisphere have reported the lowest frequencies of pulmonary radiographic abnormalities in patients with leptospirosis $[3,5,6,10]$.

The present investigation was carried out in Salvador, a large city in northeast Brazil, where there is a high incidence of leptospirosis [12]. The main 
objective was to estimate the prevalence of alterations in chest radiographs of patients hospitalized with leptospirosis and to describe the patterns of radiographic alteration in these patients.

\section{Materials and Methods}

Chest radiographs were requested for 139 patients admitted to Couto Maia Hospital, a referral center for infectious diseases, between July, 1997, to July, 1999, independent of clinical findings. These patients were participating in a randomized clinical trial developed to test the efficacy of penicillin on the late stage of leptospirosis. In keeping with the research protocol of the clinical trial, all patients were older than 15 years and had the disease with more than 4 days of symptoms. Each patient signed a consent form, approved by the local institutional review board. The radiographs were independently analyzed by two penumologists who were blinded to any other data from the patients. The agreement between the pneumologists who analized the radiograph alterations, was generally high (kappa=0.7). Radiographs with discordant diagnoses were submitted to a radiologist for independent opinion. The discordance was resolved by consensus among the three observers. The radiographic variables were: 1) presence of radiograph alterations, 2) type of abnormality: alveolar, alveolo-interstitial, interstitial and pleural effusion, 3) involved lobe, 4) side and 5) extension of the abnormalities.

The macroscopic slide test for leptospiral antigens was performed for all patients. The microagglutination test (MAT) and hemoculture for leptospira were performed for 17 and 12 patients, respectively. The probability score proposed by Faine was also used to diagnoses leptospirosis [13]. MAT and hemocultures for leptospira were performed at the Centro de Pesquisas Gonçalo Moniz da Fundação Oswaldo Cruz - FIOCRUZ. The macroscopic slide test was performed at the Laboratório Central de Saúde Pública Gonçalo Moniz - LACEN. MAT was considered positive when there was a 4-fold increase in the reciprocal titre between paired serum samples or when the reciprocal titre was greater than 800 in one or more serum samples. The macroscopic slide test was classified as positive or negative.

Statistical analysis. The statistical analysis was basically descriptive. The quantitative variables were described by the mean $( \pm \mathrm{SD})$ and the median. The categorical variables were described by their relative frequencies. Exact mid-p 95\% confidence interval for the prevalence of radiographic abnormalities was determined by using the module CONFINT of the Computer Programs for Epidemiologists, PEPI, version 3.01 [14].

\section{Results}

The diagnosis of leptospirosis was confirmed by serologic test or hemoculture in $96.4 \%$ (134/139) of the participants; in 5 pateints, the diagnosis was based on clinical and epidemiological findings according to the Faine criteria. Characteristics of the 139 patients are shown in Table 1 . The mean $( \pm \mathrm{SD})$ of age was $34.7 \pm 12.9$ years (median $=31$ years). There was a predominance of males $(87.8 \%)$; 95\% (132/139) were icteric. Dyspnea was reported by $14(10.1 \%)$ of the patients. The mean $\pm(\mathrm{SD})$ of the respiratory rate was $24 \pm 8$ movements per minute (median $=23$ ). The mean $( \pm \mathrm{SD})$ of serum creatinine was $3.9 \pm 1.9 \mathrm{mg} / \mathrm{dL}$ (median=4).

The frequency of chest radiographic alterations was $25.2 \%(35 / 139)$; $95 \% \mathrm{CI}=18.5 \%$ to $32.9 \%$ (Table 2). Among the radiographs with alterations, $74.3 \%$ (26/35) were classified as alveolar infiltrate (Figure 1 A and $1 \mathrm{~B})$. The interstitial and alveolar-interstitial types of infiltrates were found in $17.1 \%$ (6/35) and 2.8\% (1/ $35)$, respectively. The involvement was bilateral in 19 of $35(54.3 \%)$ patients. The alteration was located on the right side of the chest in 11 of the 16 pateints (68.8\%) with unilateral lesion.

Pleural effusion, represented by blunting of the costo-phrenic angle, was detected in 3 of the 35 patients $(8.6 \%)$ with abnormal radiographs. Among the 33 with parenchymatous alterations, the lesion was restricted to the inferior lobes in 15 (45.5\%). Alone, 
Table 1. Characteristics of the Patients

\begin{tabular}{lc}
\hline Characteristic & N = 139 \\
\hline Age (years) & \\
$\quad$ Mean \pm SD & $34.7 \pm 12.9$ \\
$\quad$ Median & 31 \\
Male & $122(87.8 \%)$ \\
Jaundice & $132(95 \%)$ \\
Dyspnea & $14(10.1 \%)$ \\
Respiratory Rate per Minute & \\
$\quad$ Mean \pm SD & $24 \pm 8$ \\
$\quad$ Median & 23 \\
Creatinine $(\mathrm{mg} / \mathrm{dL})$ & \\
$\quad$ Mean \pm SD & $3.9 \pm 1.9$ \\
$\quad$ Median & \\
\hline
\end{tabular}

Table 2. Distribution of pulmonary radiograph findings

\begin{tabular}{lc}
\hline \% Radiograph with Alterations & $25.2 \%(35 / 139)$ \\
(95\% CI) $\downarrow$ & $18.5 \%-32.9 \%$ \\
Type of Alteration & \\
$\%$ Alveolar Infiltrate & $74.3 \%(26 / 35)$ \\
$\%$ Interstitial Infiltrate & $17.1 \%(6 / 35)$ \\
\% Alveolo-interstitial Infiltrate & $2.8 \%(1 / 35)$ \\
\% Pleural Effusion * & $8.6 \%(3 / 35)$ \\
\hline $\begin{array}{l}\downarrow \text { CI= Confidence Interval. * A patient had alveolo-interstitial infiltrate } \\
\text { associated with pleural effusion. }\end{array}$
\end{tabular}

Figure 1. Chest radiograph of two patients with leptospirosis showing the pattern of bilateral alveolar infiltrate. A - Areas of confluent consolidation located in peripheral zones of the inferior lobes. B - Diffuse nodular and small, patchy opacities
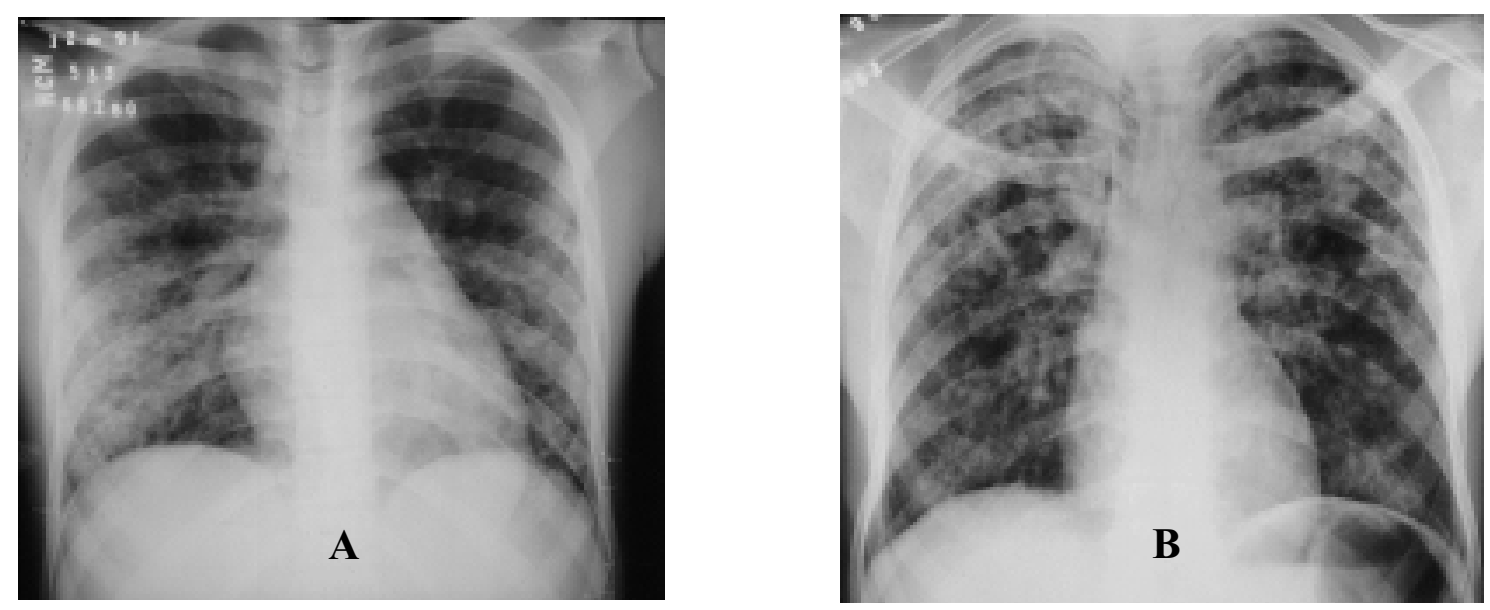
or in combination, the inferior lobes accounted for $87.9 \%$ (29/33). Involvement restricted to one or two lobes was seen in $60.6 \%(20 / 33)$ patients with parenchymatous alteration. In $15.2 \%$ (5/33), the lesion was more diffuse, involving 4 or 5 lobes.

\section{Discussion}

According to the results of the present study, pulmonary radiograph alterations can be detected in the first 48 hours after hospitalization in approximately $25 \%$ of patients older than 15 years with symptoms of leptospirosis for more than 4 days. The frequency of alterations in chest radiographs estimated in this study (25.2\%) is not very different from previous observations by other Brazilian studies, from São Paulo $(33.3 \% ; 6 / 18)$ [10] and another one from Rio de Janeiro $(34.9 \%$; 15/43) [5]. The prevalence of $25.2 \%$ found in the present study is also similar to the one described in a study from Jamaica $(22.7 \%$; 10/44) [3]. The Jamaican study is apparently the only previous investigation developed with the specific objective of estimating the prevalence of pulmonary radiograph alterations in patients with leptospirosis. A lower prevalence of pulmonary radiograph alterations (11\%) was reported by Heath, et al. in the United States [6]. Much higher, estimates of prevalence of pulmonary alterations in leptospirosis than the one found in the present study have been reported from China $(66.7 \%$; 62/93) [2]; Korea (two studies: one with $43.0 \%$ (40/93) [1], and one with 63.8\% (37/58) [11]); and Reunion Island (51.9\%, 80/154) [4].

The existing data do not allow us to conclude whether the geographic variation in pulmonary findings in the radiographic examination is related to the genetic background of the patients, environmental factors, the infecting serovars, or a combination of these factors. It is important to note, however, that the studies with the highest frequencies of alterations in the chest radiograph are those with the highest proportions of anicteric leptospirosis. In the study from China, the one with the highest prevalence of pulmonary alterations, all patients were anicteric [2]. In the Korean studies, the percentages of icteric patients were $37.0 \%$ [11] and $16 \%$ [1]. In the Reunion Island investigation, icteric patients corresponded to $47 \%$ [4]. The percentages of icteric leptospirosis in these studies (i.e., from China, Korea and Reunion Island) were lower than the percentage observed in the present study $(95 \%)$ and in the studies from São Paulo (100\%) [10] and Jamaica (68\%) [3]. Unfortunately, the percentage of icteric patients among those who had chest radiographs were not reported in the studies from Rio de Janeiro or from the United States. It is also important to note that, except for the Reunion Island study $(\mathrm{n}=154)$ [4], all of the studies had smaller sample sizes than the present one. In addition, the previous studies, except for the Jamaican study, were not planned to assess prevalence of pulmonary alterations. Thus, chance and the criteria used for radiographic indications (indication bias) should be viewed as potential explanations for the variation in the prevalence of pulmonary radiograph alterations across studies.

In the present sample, alveolar infiltrate was the most frequent pulmonary radiologic alteration. In addition, the lesions were more often bilateral. The predominance of this pattern in the radiographs has also been found in other studies [1-5, 11]. It is important to observe that alveolar infiltrate is associated with intraalveolar hemorrhage [15], a finding consistently described in autopsies and experimental studies in animals with leptospirosis [1, 2, 11, 16-18]. These findings are also consistent with evidence that the basic mechanism of pulmonary alterations in leptospirosis is a generalized capillaritis with increased susceptibility to bleeding [11, 17-19].

The relatively lower frequency of pleural effusion $(8.6 \%)$ observed in the present study is a finding also described by other investigators $[3,4,10,16]$. The highest frequencies of pleural effusion were described in two studies from Korea, with 19\% [11] and 30\% [1]. It is important to note that these Korean studies are among those with the highest prevalences of radiographic chest abnormalities overall. These data are additional support for the existence of differences in the clinical presentation of leptospirosis across geographic regions. 
The parenchymatous alterations were located more often in the inferior lobes. It should be observed, however, that lateral radiographs were not performed in the present study. Lateral radiographs are important, particularly to differentiate alterations in the inferior lobes from those in the median and the lingula lobes. It is unlikely, however, that an assessment of chest radiographs based solely on a posteroanterior projection has biased the estimate of prevalence [1-5].

According to the results from this and previous studies, radiographic chest alterations are relatively frequent in patients with leptospirosis. These alterations are more often bilateral and consistent with alveolar infiltrates. Studies are still necessary to describe the evolution of the pulmonary findings in patients with leptospirosis. It is also important to assess the value of alveolar infiltrates in the identification of cases of leptospirosis among patients with different forms of the disease. The determinants of the large variation in the prevalence of radiographic chest alterations in patients with leptospirosis across geographic regions is another important question for future research.

\section{References}

1. Park S.K., Lee S.H., Rhee Y.K., et al. Leptospirosis in Chonbuk Province of Korea in 1987: a study of 93 patients. Am J Trop Med Hyg 1989;41:345-51.

2. Wang C., Ch'i C., Lu F. Studies on anicteric leptospirosis. III. Roentgenologic observations of pulmonary changes. Chin Med J 1965;84:293-306.

3. Lee R.E., Terry S.I., Walker T.M., Urquhart A.E. The chest radiograph in leptospirosis in Jamaica. Br J Radiol 1981; 54:939-43.

4. Courtin J., Di Francia M., Poubeau P., et al. Les manifestations respiratoires de la leptospirosis. Étude rétrospective de 91 cas (1978-1994). Rev Pneumol Clin 1998;54:382-92.

5. Mascarenhas L., Gonçalves A., Cunha R., et al. Manifestações respiratórias na leptospirose. Arq Bras Med 1991;65:49-51.

6. Heath C., Alexander A., Galton M. Leptospirosis in the United States. Analysis of 483 cases in man, 1949-1961. N England J Med 1965;273:857-64.

7. Ko A., Reis M., Dourado C., et al. Urban epidemic of severe leptospirosis. Lancet 1999;354:820-25.
8. Pereira M.M., Andrade J. Human leptospirosis in a slum area in the city of Rio de Janeiro, Brazil—a serological and epidemiological study. Mem Inst Oswaldo Cruz 1990; $85: 47-52$.

9. Farr R. Leptospirosis. Clin Infect Dis 1995;21:1-6.

10. Nery L.E., de Paula A.B., Nakatani J., et al. Clinical, radiological and functional pulmonary manifestations in patients with leptospirosis. Rev Inst Med Trop Sao Paulo 1977; 19:366-73.

11. Im J.G., Yeon K.M., Han M.C., et al. Leptospirosis of the lung: radiographic findings in 58 patients. AJR Am J Roentgenol 1989;152:955-9.

12. Secretaria de Saúde do Estado da Bahia. Relatório da Avaliação Epidemiológica da Leptospirose no Estado da Bahia. Salvador (Bahia,Brazil): Departamento de Vigilância da Saúde, 1996.

13. Faine S. Guidelines for the control of leptospirosis. WHO Offset Publ 1982;67:1-171.

14. Abramson J.H., Gahlinger P.M. Computer Programs for Epidemiologists: PEPI. Vol. USD Inc. Stone Mountain, GA, 1999.

15. Green R.J., Ruoss S.J., Kraft S.A., et al. Pulmonary capillaritis and alveolar hemorrhage. Update on diagnosis and management. Chest 1996;110:1305-16.

16. Silverstein C. Pulmonary Manifestations of Leptospirosis. Radiology 1953;61:327-33.

17. De Brito T., Bohm G.M., Yasuda P.H. Vascular damage in acute experimental leptospirosis of the guinea-pig. J Pathol 1979; 128:177-82.

18. Nicodemo A.C., Duarte M.I., Alves V.A., et al. Lung lesions in human leptospirosis: microscopic, immunohistochemical, and ultrastructural features related to thrombocytopenia. Am J Trop Med Hyg 1997;56:181-7.

19. Miller N.G., Allen J.E., Wilson R.B. The pathogenesis of hemorrhage in the lung of the hamster during acute leptospirosis. Med Microbiol Immunol 1974;160:269-78. 